\title{
Le froid en fromagerie de chèvre(1)
}

\author{
par \\ B. TIERSONNIER \\ Ingénieur en Chef du Génie Rural des Eaux et des Forêts
}

La production annuelle de lait de chèvre, à peu près stable ces deux dernières années, est de $280000 \mathrm{hl}$ environ, soit très approximativement le $1 / 100$ de celle du lait de vache. Les statistiques indiquent, d'autre part, que les $3 / 4$ de cette production sont obtenus dans trois Régions :

\begin{tabular}{lr} 
Poitou-Charente & $800000 \mathrm{hl}$ \\
Rhône-Alpes & $775000 \mathrm{hl}$ \\
Centre & $500000 \mathrm{hl}$ \\
\cline { 2 - 2 } & $2075000 \mathrm{hl}$
\end{tabular}

Cette répartition est d'ailleurs peu significative car elle ne traduit pas le caractère local d'une production qui est en fait concentrée dans des terroirs relativement peu étendus et marquant fortement la fabrication de fromage qui fait leur réputation.

La fromagerie de chèvre produit $28000 \mathrm{t}$ par an, tonnage modeste vis-à-vis des $680000 \mathrm{t}$ des fromageries de lait de vache ; néanmoins c'est une spéculation intéressante localement par le revenu qu'elle procure aux agriculteurs, car la demande est soutenue et la vente rémunératrice. Globalement, il faut environ 71 de lait de chèvre pour $1 \mathrm{~kg}$ de fromage, mais des variations assez importantes interviennent suivant les types de fabrication.

La fromagerie de chèvre est restée artisanale jusqu'à une date récente ; les fromages fabriqués à la ferme étaient ramassés par des affineurs dont les pratiques ancestrales jointes aux conditions du terroir aboutissaient en général à des produits de grande qualité, quoique irréguliers car les conditions naturelles de l'affinage dépendent par trop des conditions extérieures.

La fromagerie de chèvre en est donc venue, au moins partiellement, à s'industrialiser en régularisant son rythme de fabrication au long de l'année, et d'autre part en recréant artificiellement l'ambiance

(1) Génie Rural, 1969, $\mathrm{n}^{\circ} 6$. 
propice à l'affinage. Cette évolution vers la rationalisation de la fromagerie est devenue possible en faisant appel aux techniques du froid, essentiellement : la congélation du caillé en saison de haute production et le conditionnement de l'air des locaux d'affinage.

Dans ce qui suit, nous prendrons pour exemple la fabrication dans une Coopérative des bords de la Loire des fromages de chèvre du Sancerrois, bien connus sous le nom de " crottins de Chavignol ", petits fromages ronds, aplatis, consommés très secs.

\section{Aperçu sur la fabrication}

Le schéma de fabrication est rappelé ci-dessous :

- Quantité de lait : 0,55 à 0,65 1 par fromage.

- Caillage lent : 20 à $24 \mathrm{~h}$, obtenu par adjonction de souches de ferments lactiques et d'un peu de présure; autrement dit : caillage naturel, mais pâte rendue un peu plus ferme grâce à la présure.

- Egouttage : $24 \mathrm{~h}$ dans des poches en toile.

- Moulage : dans des moules en grès perforés où l'égouttage s'achève ; durée $24 \mathrm{~h}$.

- Salage : après démoulage, les fromages sont successivement salés au sel fin sur une face, retournés, salés sur l'autre face et sur les côtés ; durée 24 h.

- A la fin du salage, le poids d'un fromage est de $160 \mathrm{~g}$.

- Hâlage et affinage : durée $12 \mathrm{j}$; perte de poids : $80 \mathrm{~g}$, soit environ la moitié du poids du fromage frais rentrant dans le hâloir.

Indépendamment du processus de fabrication et d'affinage, la qualité des fromages dépend de celle du lait de chèvre dont la propreté bactériologique est indispensable ; le lait est par suite ramassé tous les jours et immédiatement traité.

L'usine a une capacité de fabrication de 5000 fromages par jour ; de mai en août, la production laitière est nettement excédentaire et une partie du caillé, après égouttage, est congelée, puis stockée à $-20^{\circ} \mathrm{C}$ pour être reprise en fabrication pendant les mois d'automne et d'hiver.

\section{Congélation et utilisation du caillé congelé}

La congélation du caillé de chèvre est une pratique déjà très répandue dans le Centre et en Poitou; les stocks de caillé congelé représentent d'ailleurs une fraction non négligeable de l'activité des entrepôts frigorifiques publics régionaux, et chaque année des installations spécialisées se réalisent pour la congélation et la conservation du caillé de chèvre dans les usines situées à l'écart des zones d'action 
des entrepôts. On connaît donc déjà, au moins qualitativement, les conditions nécessaires à une bonne congélation du caillé.

Les essais entrepris au Laboratoire de l'Ecole Laitière de Surgères (Charente-Maritime), et amplement confirmés au plan industriel, montrent que la qualité initiale du caillé doit être excellente : traitement rapide après la collecte, propreté des toiles et sacs d'égouttage, égouttage convenablement réglé de façon à ce que l'extrait sec du caillé soit de 38 à $40 \mathrm{~g}$ pour $100 \mathrm{~g}$.

La rapidité de la congélation est un autre facteur de la qualité du produit conservé. En pratique, le caillé est emballé dans des sacs en film plastique de $20 \mathrm{~kg}$, placés dans des moules en aluminium de 12 à $15 \mathrm{~cm}$ de hauteur, puis introduits dans un tunnel de congélation à $-35^{\circ} \mathrm{C} /-40^{\circ} \mathrm{C}$ où il séjourne de 16 à $24 \mathrm{~h}$, temps au bout duquel la température à cour est au plus de $-15^{\circ} \mathrm{C}$. Les tunnels spécialisés, réalisés en 1968, ont une capacité unitaire de 3 à 5 t de caillé ; ils sont alimentés au R 502 et la température d'évaporation est de $-45^{\circ} \mathrm{C}$.

La congélation lente en chambre ventilée à $-25^{\circ} \mathrm{C}$, n'est pas recommandée; les fabrications faites à partir de caillé congelé de cette façon sont, en effet, de moins bonne qualité qu'en congélation rapide en tunnel.

La température optima de conservation du caillé congelé n'est pas encore déterminée avec certitude ; un stockage entre -20 et $-25^{\circ} \mathrm{C}$ semble assez satisfaisant pour des caillés préparés à partir de lait de bonne qualité bactériologique et dans des conditions conformes à ce qui a été dit plus haut. Il importe également que la température de stockage soit constante, car on a remarqué dans les chambres où s'étaient produites des remontées de température, une qualité moins bonne des fromages.

Là où les conditions précédentes sont bien remplies, la durée de stockage du caillé peut atteindre 6 mois sans qu'on observe ultérieurement de défauts de fabrication ; du moins dans la plupart des cas, car certaines anomalies, dont la cause n'est pas encore connue, ont toutefois été relevées sur des lots de caillé après 3 mois de stockage, se traduisant par un goût d'oxydé plus ou moins accentué.

En conclusion, la congélation du caillé de chèvre obéit aux règles générales de la congélation : qualité initiale du produit, rapidité de l'opération, stockage à température suffisamment basse et constante ; la particularité à noter est l'exigence d'une température inférieure à $-20^{\circ} \mathrm{C}$, sensiblement plus basse que celle requise par d'autres produits (viande bovine, beurre) pour une durée de conservation ne dépassant guère 6 mois.

Au moment de reprendre le processus de fabrication, le caillé doit être décongelé. La décongélation sera lente, autrement dit se faire à l'air à une température de l'ordre de $+10^{\circ} \mathrm{C}$. A la Coopérative précitée du Val-de-Loire, la décongélation est faite en deux 
temps : 5 jours à l'air à $+6^{\circ} \mathrm{C}$, puis 1 ou 2 jours à température plus élevée $\left(+12^{\circ}\right.$ à $\left.+15^{\circ} \mathrm{C}\right)$. La décongélation rapide en plongeant dans de l'eau courante les sacs de caillé est à écarter.

L'utilisation du caillé décongelé nécessite quelques précautions; il ne doit pas être employé pur, car la texture du fromage tendrait à devenir granuleuse ou friable ; on le mélangera, par conséquent, à du caillé frais dans une proportion telle que l'extrait sec du mélange soit le même que celui du caillé frais. Moyennant cette précaution, il n'y aura pas lieu de changer les conditions au hâloir.

\section{Hâlage et affinage}

Les fromages de Chavignol séjournent au moins 12 jours au hâloir ; de $160 \mathrm{~g}$ leur poids revient à $80 \mathrm{~g}$. On perd donc $80 \mathrm{~g}$ d'eau pendant ce laps de temps dont :

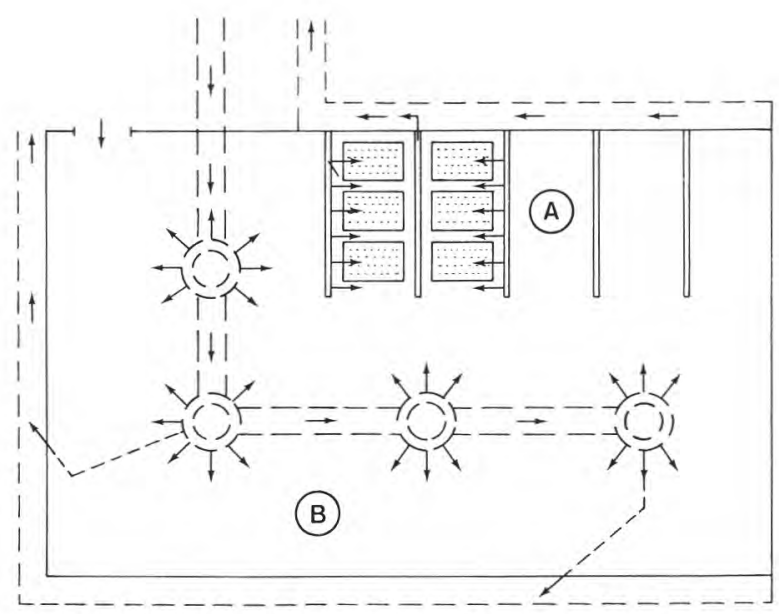

SALLE DE HALAGE-AFFINAGE

A : Cellule de halage

B: ZONE D'AFFINAGE

- 60 g doivent être évacués au cours des 5 premiers jours ; c'est le hâlage proprement dit, et

- $20 \mathrm{~g}$ au cours des 7 jours suivants : affinage minimum avant commercialisation.

La température du hâloir doit être de $+14^{\circ} \mathrm{C}$ et le degré hygrométrique de 75 à 80 p. 100, ambiance dans laquelle le fromage perdra en 5 jours le $1 / 3$ de son poids d'eau. Ce pompage d'humidité, facilité il est vrai par la petite dimension des fromages, est considérable ; 
il implique une ventilation énergique, car on ne peut guère entraîner plus de $1 \mathrm{~g}$ d'eau par kg d'air en circulation et, d'autre part, discontinue, ne couvrant que $16 \mathrm{~h}$ par jour de façon à permettre la migration de l'humidité du centre du fromage vers sa périphérie.

Nous ne nous étendrons pas sur les calculs de climatisation qui restent dans le cadre classique des hâloirs, nous bornant simplement à donner leurs résultats et les caractéristiques d'équipement.

Volume du hâloir ............ $560 \mathrm{~m}^{3}$

Capacité totale ............. 60000 fromages (ou 9,5 t)

Puissance frigorifique installée .. $80000 \mathrm{fr} / \mathrm{h}\left(0^{\circ} \mathrm{C}+25^{\circ} \mathrm{C}\right)$

Puissance calorifique ......... $56000 \mathrm{Kcal} / \mathrm{h}$

Débit d'air en circulation ....... $46000 \mathrm{~kg} / \mathrm{h}$ (ou $37000 \mathrm{~m}^{3} / \mathrm{h}$ )

On note l'importance des puissances frigorifique et calorifique installées ainsi que le débit d'air.

L'originalité de l'installation coopérative examinée réside dans son aménagement : le hâloir et la salle d'affinage sont, en effet, confondus en un seul local dont le schéma est donné ci-contre.

D'une part : 5 cellules, en forme de tunnel, délimitées par des murs creux, à paroi soufflante, chaque tunnel correspondant à la production journalière de 5000 fromages; ceux-ci rangés sur des étagères roulantes séjournent 5 jours dans le tunnel. La ventilation est faite alternativement d'un mur creux vers l'autre, puis inversement ; l'air conditionné est soufflé horizontalement au niveau même des plateaux des étagères et l'alternance du sens de soufflage procure une bonne homogénéité du hâlage.

D'autre part : le reste de la salle, qui sert de salle d'affinage et dans laquelle stationnent pendant une semaine les étagères retirées du hâlage. L'air conditionné est distribué par diffuseurs-plafonniers et repris par gaines encastrées dans les plinthes. La ventilation est beaucoup moins intense dans la zone « affinage » que dans les cellules de hâlage.

Cette disposition procure, en outre, une bonne commodité pour les manutentions qui deviennent assez réduites.

D'autres dispositions sont évidemment possibles, choisies notamment en fonction des particularités de l'affinage après hâlage ; il n'est pas douteux que cette substitution de méthodes artificielles aux méthodes naturelles permette d'obtenir une bonne qualité permanente de fabrication, sans bien entendu prétendre aux qualités supérieures obtenues de temps à autre par les méthodes de l'empirisme. 